\title{
Audiovisual biofeedback breathing guidance for lung cancer patients receiving radiotherapy: a multi-institutional phase II randomised clinical trial
}

Sean Pollock ${ }^{1 *}$, Ricky O'Brien ${ }^{1}$, Kuldeep Makhija ${ }^{1}$, Fiona Hegi-Johnson², Jane Ludbrook ${ }^{3}$, Angela Rezo ${ }^{4}$, Regina Tse ${ }^{5}$, Thomas Eade ${ }^{6}$, Roland Yeghiaian-Alvandi ${ }^{7,8}$, Val Gebski ${ }^{9}$ and Paul J Keall ${ }^{1}$

\begin{abstract}
Background: There is a clear link between irregular breathing and errors in medical imaging and radiation treatment. The audiovisual biofeedback system is an advanced form of respiratory guidance that has previously demonstrated to facilitate regular patient breathing. The clinical benefits of audiovisual biofeedback will be investigated in an upcoming multi-institutional, randomised, and stratified clinical trial recruiting a total of 75 lung cancer patients undergoing radiation therapy.

Methods/Design: To comprehensively perform a clinical evaluation of the audiovisual biofeedback system, a multi-institutional study will be performed. Our methodological framework will be based on the widely used Technology Acceptance Model, which gives qualitative scales for two specific variables, perceived usefulness and perceived ease of use, which are fundamental determinants for user acceptance. A total of 75 lung cancer patients will be recruited across seven radiation oncology departments across Australia. Patients will be randomised in a 2:1 ratio, with $2 / 3$ of the patients being recruited into the intervention arm and 1/3 in the control arm. 2:1 randomisation is appropriate as within the interventional arm there is a screening procedure where only patients whose breathing is more regular with audiovisual biofeedback will continue to use this system for their imaging and treatment procedures. Patients within the intervention arm whose free breathing is more regular than audiovisual biofeedback in the screen procedure will remain in the intervention arm of the study but their imaging and treatment procedures will be performed without audiovisual biofeedback. Patients will also be stratified by treating institution and for treatment intent (palliative vs. radical) to ensure similar balance in the arms across the sites. Patients and hospital staff operating the audiovisual biofeedback system will complete questionnaires to assess their experience with audiovisual biofeedback. The objectives of this clinical trial is to assess the impact of audiovisual biofeedback on breathing motion, the patient experience and clinical confidence in the system, clinical workflow, treatment margins, and toxicity outcomes.

(Continued on next page)
\end{abstract}

\footnotetext{
* Correspondence: sean.pollock@sydney.edu.au

1 Radiation Physics Laboratory, Sydney Medical School, The University of

Sydney, Sydney, NSW, Australia

Full list of author information is available at the end of the article
} 
(Continued from previous page)

Discussion: This clinical trial marks an important milestone in breathing guidance studies as it will be the first randomised, controlled trial providing the most comprehensive evaluation of the clinical impact of breathing guidance on cancer radiation therapy to date. This study is powered to determine the impact of AV biofeedback on breathing regularity and medical image quality. Objectives such as determining the indications and contraindications for the use of AV biofeedback, evaluation of patient experience, radiation toxicity occurrence and severity, and clinician confidence will shed light on the design of future phase III clinical trials.

Trial registration: This trial has been registered with the Australian New Zealand Clinical Trials Registry (ANZCTR), its trial ID is ACTRN12613001177741.

Keywords: Breathing guidance, Motion management, Randomised, Stratified, Phase II clinical trial, Lung cancer, Radiotherapy

\section{Background}

The precision of radiotherapy can be reduced due to respiratory-related tumour motion, particularly for tumours in the thoracic region, leading to increased irradiation of healthy surrounding tissues, resulting in a significant increase in radiation-related toxicity [1-3]. This is further exacerbated when respiration is irregular in nature (deep/shallow breaths, baseline shifts, suspended breathing, etc.) $[4,5]$. A 1 Gy increase in tumour dose results in a $4 \%$ improvement in survival, [6] however, a $0.5 \mathrm{~cm}$ range of tumour motion can cause a $4 \sim$ $5 \%$ variation in radiation dose [7] which leads to an increase in mean dose to healthy surrounding tissues resulting in an increase in risk of pneumonitis and radiation toxicity $[8,9]$.

Techniques such as respiratory gating, breath-holds and tumour tracking are clinically useful for tumour motion management $[10,4,11]$. However, irregular respiration can reduce the efficiency of such motion management techniques, $[12,13]$ irregular respiration also causes motion artefacts and anatomic errors in medical imaging [14-19].

Breathing guidance is one such technique which specifically aims to produce regular patient breathing by showing the patient how to adjust their breathing in real-time. One such breathing guidance system is the audiovisual (AV) biofeedback system (shown in Fig. 1), developed by Venkat, et al [13].
AV biofeedback is a real-time, interactive and personalised respiratory guide designed to facilitate regular patient breathing. Table 1 outlines the findings from previous AV biofeedback investigations.

However, none of the studies presented in Table 1 were randomised trials, in addition to this, the findings of a recent literature search yielded that a randomised clinical trial with any breathing guidance intervention has not yet been performed. To fill the gap in the literature, we have designed a multi-institutional, phase II, randomised clinical trial to thoroughly assess the clinical impact of the AV biofeedback breathing guidance system. Based on previous findings, we hypothesise that AV biofeedback will significantly improve breathing regularity and reduce medical imaging errors for lung cancer patients undergoing imaging and treatment procedures during radiotherapy.

This trial has been registered with the Australian New Zealand Clinical Trials Registry (ANZCTR), its trial ID is ACTRN12613001177741.

\section{Methods/Design}

This study aims to assess the clinical impact of AV biofeedback by recruiting 75 lung cancer patients across seven radiation oncology departments. What follows is an outline of the AV biofeedback setup, primary and secondary objectives, participant selection criteria, the study workflow, and statistical considerations for our study design.

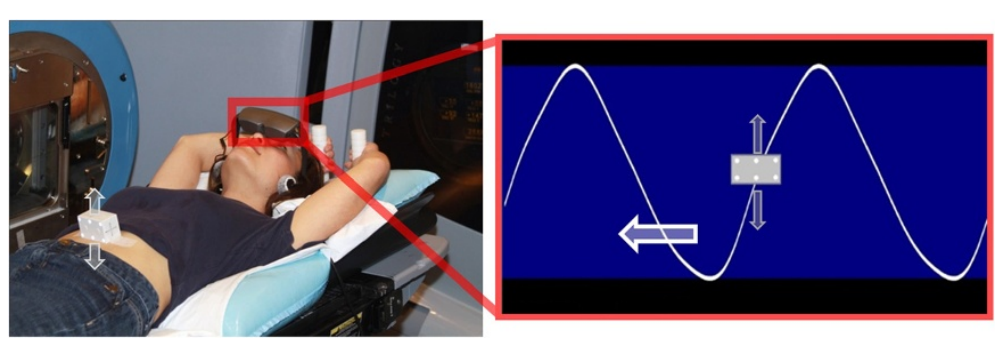

Fig. 1 AV biofeedback system (left). Display goggles and real-time position management (RPM) marker block on the abdomen shown. The visual display (right), as seen by the patient, of the AV biofeedback guiding interface shows the waveguide (white curve) and a marker position (grey marker) in real time 
Table 1 Details of previous AV biofeedback investigations

\begin{tabular}{|c|c|c|}
\hline Investigation author (Year) & Participants & Findings \\
\hline George [23] (2006) & 24 lung cancer patients & - Residual breathing motion within a gating window improved \\
\hline Venkat [13] (2008) & 10 healthy volunteers & $\begin{array}{l}\text { - Waveguide breathing guidance produced more regular breathing } \\
\text { than bar-model guidance and free breathing }\end{array}$ \\
\hline Yang [22] (2012) & Phantom study & - 4D PET image quality improved \\
\hline \multirow[t]{2}{*}{ An [36] (2013) } & \multirow{2}{*}{$\begin{array}{l}\text { Retrospective analysis } \\
\text { of George (2006) data }\end{array}$} & - CTV coverage improved \\
\hline & & • Internal motion variation improved \\
\hline \multirow{3}{*}{$\begin{array}{l}\text { Kim, [21] Pollock, [37] \& } \\
\text { Steel [38] (2012-2014) }\end{array}$} & \multirow[t]{3}{*}{15 healthy volunteers } & - Kim (2012): Breathing regularity of thoracic diaphragm and abdominal wall improved \\
\hline & & - Pollock (2013): Accuracy of kernel density estimation motion prediction improved \\
\hline & & $\begin{array}{l}\text { - Steel (2014): Strong correlation between internal and external anatomic motion } \\
\text { for both AV biofeedback and free breathing }\end{array}$ \\
\hline \multirow[t]{2}{*}{ Lee [24] (2014) } & \multirow[t]{2}{*}{5 healthy volunteers } & - Improved 3D MR image quality \\
\hline & & - Reduced gated MRI scan time \\
\hline \multirow[t]{2}{*}{ Lu [39] (2014) } & \multirow[t]{2}{*}{13 lung \& liver cancer patients } & - Breathing regularity improved \\
\hline & & - ITV $\mathrm{MIP}_{\mathrm{P}}$ underestimated ITV 10 \\
\hline \multirow[t]{2}{*}{ Lee [40] (2014) } & \multirow[t]{2}{*}{7 lung cancer patients } & - Improved intrafraction lung tumour motion consistency \\
\hline & & - Improved interfraction lung tumour motion consistency \\
\hline
\end{tabular}

\section{Research Ethics Committee}

The protocol for this clinical trial has been reviewed and approved by the Hunter New England Human Research Ethics Committee (HREC). This Human Research Ethics Committee is constituted and operates in accordance with the National Health and Medical Research Council's 'National Statement on Ethical Conduct in Human Research (2007)' (National Statement) and the 'CPMP/ICH Note of Guidance on Good Clinical Practice'. The Hunter new England HREC has also been accredited by the New South Wales Department of Health as a lead HREC under the single ethical and scientific review. A report on the progress of this clinical trial is required to be submitted annually to the Hunter New England HREC.

\section{Audiovisual biofeedback system}

The AV biofeedback system, as shown in Fig. 1, utilises the Real-time Position Management system (RPM, Varian Medical Systems, Palo Alto, USA) to track the motion of an external marker positioned on the patient's abdomen. This real-time respiratory-motion is used by the AV biofeedback software to calculate an average cycle of respiration (using a Fourier series fit from 10 obtained respiratory cycles). This average cycle is used as the waveguide (white curve in Fig. 1) which continually moves from right-to-left across the visual display and acts as part of the visual prompt for AV biofeedback. Also on the visual display is a grey marker moving vertically up-anddown corresponding to the anterior-posterior motion of the marker block positioned on the patent's abdomen. It is the goal for the patient to keep the marker block within inhale-exhale limits (presented as the blue region in Fig. 1) and match the grey marker block over the white waveguide. The audio component of AV biofeedback is classical music playing to the patient; the music fades to silence should the marker block move outside the blue area breathing limits. AV biofeedback has been shown to be compatible in a number of imaging and treatment modalities, [20-22] as well as utilising different types of patient displays [23, 21, 24]. There are two options for patient display in this study: video goggles, or a screen mounted to the couch. Which patient display option is utilised in this study will depend on what is available at each institution.

Figure 2 illustrates the schematic of the AV biofeedback study setup, from the RPM camera monitoring patient breathing motion, to the AV biofeedback computer receiving the RPM signal and extending the AV biofeedback guiding interface to the patient display.

\section{Objectives}

This clinical trial will recruit 75 lung cancer patients across 7 radiation oncology departments testing the following objectives:

Primary objective: In a prospective multi-institutional randomised clinical trial we will test the hypothesis that AV biofeedback will significantly improve breathing regularity and reduce medical imaging errors for lung cancer patients undergoing imaging and treatment procedures during radiotherapy.

Secondary objectives will involve patient-specific and department-specific objectives: 


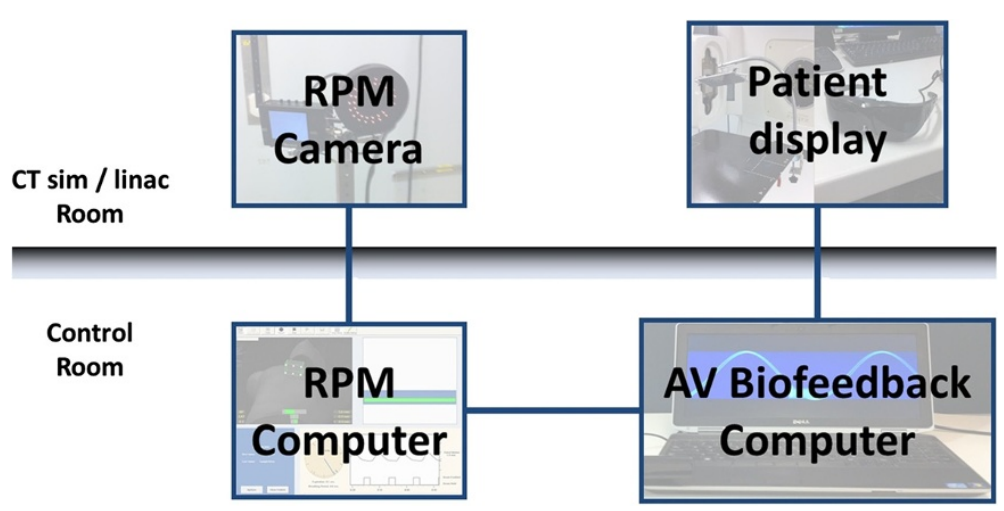

Fig. 2 Audiovisual biofeedback study setup schematic

Patient-specific objectives are to evaluate the impact of AV biofeedback by:

1) Quantifying the proportion of patients for whom breathing is more regular with AV biofeedback,

2) Quantifying the variability in breathing motion throughout a course of treatment,

3) Quantifying the improvement in image quality with AV biofeedback,

4) Evaluating the patient experience through a perception of care survey,

5) Developing indications and contra-indications for the use of AV biofeedback,

6) Quantifying the differences in image-guided radiotherapy (IGRT) shifts during treatment, and

7) Recording toxicity outcomes for up to 12 months after treatment has been completed.

Department-specific objectives are to evaluate the impact of AV biofeedback on clinical testing by:

1) Quantifying any practice changes (e.g. margin reduction),

2) Quantifying the impact on workflow using the AV biofeedback device through time-motion studies,

3) Evaluating the operator and clinician confidence in the AV biofeedback device's reliability and clinical efficacy through a technology-impact survey,

4) Quantifying the system robustness through hardware and software fault reporting, and

5) Performing system quality assurance, sharing the results through web-based uploads and provide feedback for QA improvement.

Our methodological framework will be based on the widely used Technology Acceptance Model (TAM) $[25,26]$. The TAM gives qualitative scales for two specific variables, perceived usefulness and perceived ease of use, which are fundamental determinants for user acceptance.

\section{Study participant selection criteria}

This study will recruit patients with cancer of the lung receiving external beam radiation therapy. Patients fitting the eligibility criteria (see below) will be identified and introduced to this study by their treating physicians, who will participate as investigators in this study. The eligibility criteria are as follows:

1) Lung cancer patients

i. No restrictions to type of external beam radiation therapy being received

ii. Primary or secondary cancer

2) $>18$ years old

3) No gender or ethnic restrictions

4) An ECOG score in the range of 0 to 2

5) Able to give written informed consent and willingness to participate and comply with the study

6) No pregnant / lactating woman

\section{Study workflow}

Once informed consent has been obtained, the patient will be randomised into either the intervention or control arm of the study. For patients randomised into the intervention arm, prior to their planning and treatment they will undergo a breathing decision session during which they will breathe both with and without the guidance of AV biofeedback. Preceding each breathing session will be a training session to familiarise the patient with the AV biofeedback system. After the breathing decision session has been completed, the most reproducible breathing condition (AV biofeedback or free breathing) will be determined in situ by an 'Analyse Respiratory Session' function within the AV biofeedback software. It will be the most reproducible breathing condition that will continue to be used throughout the rest of that particular patient's planning and treatment. The flowchart for this study is shown in Fig. 3.

For all patients, each follow-up visitation they have with their treating physician for the first 12 months after their treatment has finished, their treating physician will 


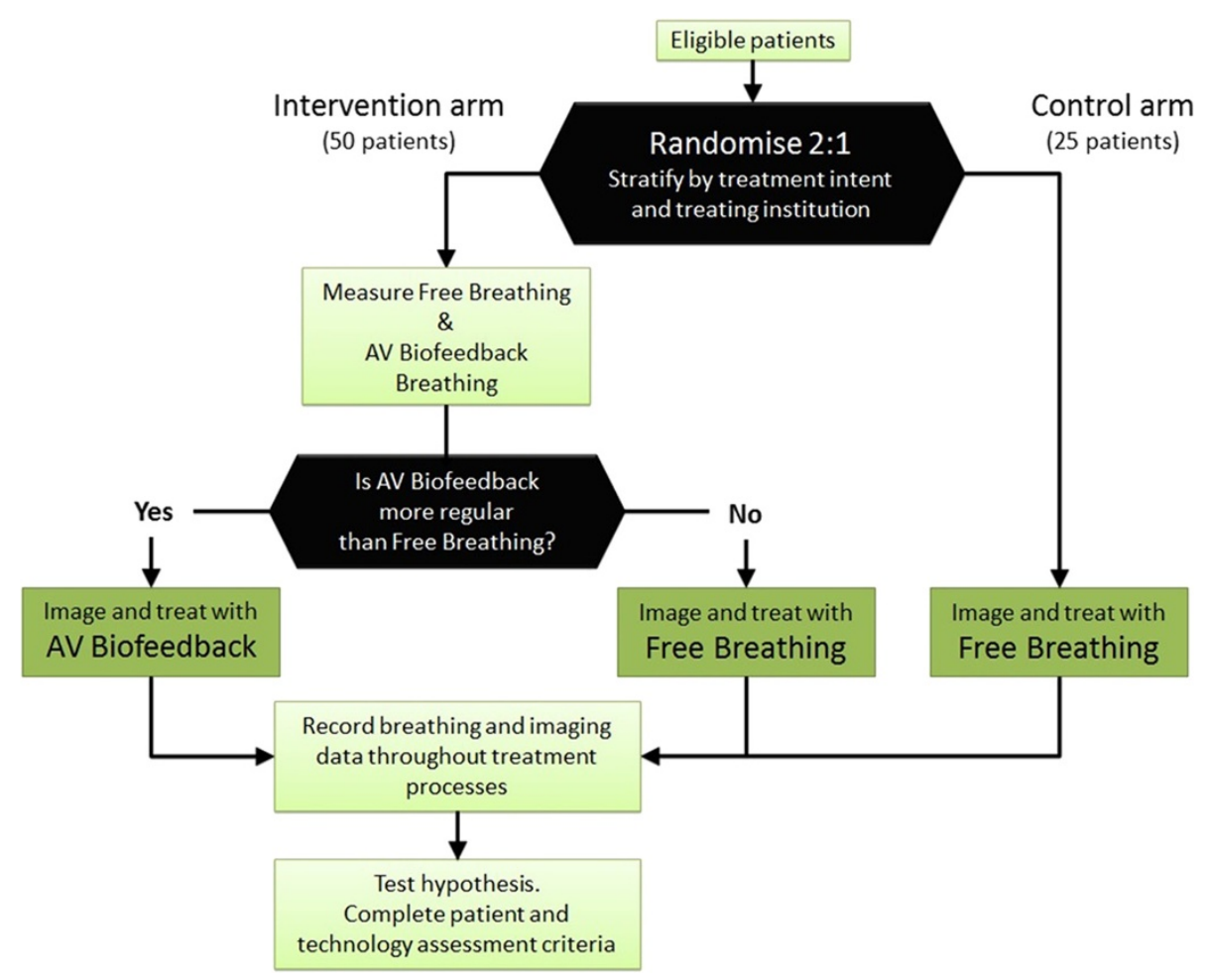

Fig. 3 Study flowchart

complete a toxicity report to satisfy the Secondary Patient-Specific Objective 7: Recording toxicity outcomes for up to 12 months after treatment has been completed by reporting the occurrence and severity of any radiation toxicities.

\section{Patient randomisation}

This trial is stratified, hence, study group random allocation will be determined by minimisation [27, 28]. Patients will be stratified by treating institution and for treatment intent (palliative vs. radical) and minimisation considerably reduces the imbalance of these stratification factors across the control and intervention groups of the study. Patients will be randomised in a 2:1 ratio, 2 out of 3 patients will be randomised into the AV biofeedback (intervention) arm and 1 out of 3 will be randomised into the free breathing (control) arm as illustrated by Fig. 3 .

\section{Sample size and power calculation}

The statistical considerations for this study are largely based on a previous study conducted at Virginia Commonwealth University (VCU) on 24 lung cancer patients $[23,29]$. Prior to this multi-institutional clinical trial, the VCU study was the largest AV biofeedback investigation, recruiting a total of 26 lung cancer patients, however, 2 patients dropped out due to not being treated with radiotherapy or rapid worsening of disease, and so their data was not collected. In the VCU study 109 breathing sessions were performed comparing AV biofeedback to free breathing, of which, 87 sessions (80 \%) demonstrated more regular breathing with AV biofeedback. Framing this is in a more clinical relevant way: irregular breathing motion exacerbates the systematic errors $(\Sigma)$ arising from motion image artefacts and variations between the planned and treated anatomy, as well as random errors $(\sigma)$ from day-to-day variations in the treated anatomy [30, 15, 31]. To combine systematic and random errors and estimate the margin contribution due to breathing irregularity we will use the van Herk method [32]: margin $=2.5 \Sigma+0.7 \sigma$, incorporating the respiratory components of systematic and random errors. A clinically significant difference in clinical improvement due to AV biofeedback has been determined to be a margin calculation of less than $5 \mathrm{~mm}$. This magnitude of reduction was elected as clinically significant because this is the same magnitude of displacement attributed to contributing to significant artefacts and errors during radiotherapy procedures as detailed in AAPM Task Group 76 [4]. From this van Herk calculation, in the VCU study there were $14 / 24$ patients with margins $<5 \mathrm{~mm}$ with AV biofeedback, while only $5 / 24$ for free breathing.

In this proposed study, to get a more accurate indication of the proportion of patients with reduced margins calculated using the van Herk method we have designed 
an exploratory phase II randomised study examining the potential impact of an AV biofeedback system in regulating breathing in patients receiving radiation therapy for the treatment of lung cancer. Without the AV biofeedback system, it is conservatively estimated that approximately $40 \%$ of patients naturally exhibit regular breathing (margin component below $5 \mathrm{~mm}$ ). Increasing this proportion to $60 \%$ using the AV biofeedback system would be clinically worthwhile. Based on Simon's design, [33] a sample size of 50 patients receiving the AV biofeedback system will have at least $80 \%$ power with $95 \%$ confidence to rule out a regular rate of $40 \%$ in favour of a $60 \%$ rate. To minimise patient selection bias and provide an estimate of regular breathing from a contemporary control, the proposed design will be a randomised phase II with a 50 patients receiving the intervention and 25 receiving current standard of care. Patients will be randomised in a 2:1 ratio, with $2 / 3$ of the patients being recruited into the AV biofeedback (intervention) arm and $1 / 3$ in the free breathing (control) arm as illustrated by Fig. 3. 2:1 randomisation is appropriate as within the interventional arm there is a screening procedure where only patients whose breathing is more regular with AV biofeedback use this system for their imaging and treatment procedures. Patients will be stratified by treating institution and for treatment intent (palliative vs. radical) to ensure similar balance in the arms across the sites. As the study is not powered for formal comparisons between the groups, estimates of the proportion of patients which do not experience irregular breathing will provide information as to whether further investigation is warranted.

Assuming a contamination and dropout rate of no more than $10 \%$, this study will require that $75+8=83$ patients be recruited (the $10 \%$ value was based on the $2 / 26$ patient drop-out rate in the VCU study).

Patients at each institution will be treated per department protocol with no additional constraints on dose, fractionation, immobilisation or image guided procedures.
Results will be adjusted for institution (using a fixed effect) to account for differences between institutions.

\section{Data analysis}

The primary objective is to assess the impact of AV biofeedback on breathing regularity and image errors; the section that follows details the metrics to be utilised for the primary objective.

Breathing motion regularity is quantified as the root mean square error (RMSE) in displacement and period $[13,21,24,34]$. A breathing signal is separated into its individual cycles and an 'average' waveform is calculated using a Fourier series fit. Figure 4 illustrates an example breathing trace, its separation into cycles, and its average waveform.

RMSE will be calculated as detailed by Venkat, et al., (2008),[13] but will be outlined here for clarity. For a breathing pattern comprised of $n$ individual breathing cycles, where each cycle in the phase domain can be written as $X=\left\{x_{1}, x_{2}, \ldots, x_{360}\right\}$ and the average waveform of these cycles can be written as $Y=\left\{y_{1}, y_{2}, \ldots, y_{360}\right\}$, the RMSE in displacement is calculated as:

$$
\text { RMSE in displacement }=\frac{\sum_{\text {All Cycles }} \sqrt{\sum_{i=1 \ldots 360} \frac{\left(x_{i}-y_{i}\right)^{2}}{360}}}{n}
$$

The period of each of the $n$ breathing cycles, in seconds, can be written as $P=\left\{p_{1}, p_{2}, \ldots, p_{n}\right\}$, with the period of the average waveform expressed as Period ${ }_{\text {mean }}$, the RMSE in period is calculated as:

$$
\text { RMSE in period }=\sqrt{\frac{\sum_{i=1 \ldots n}\left(p_{i}-\text { Period }_{\text {mean }}\right)^{2}}{n}}
$$

The impact of AV biofeedback on 4D-CT image quality will utilise an automated method of image artefact identification developed by Cui, et al., (2012), [35] but will be outlined here for clarity. The method is based on
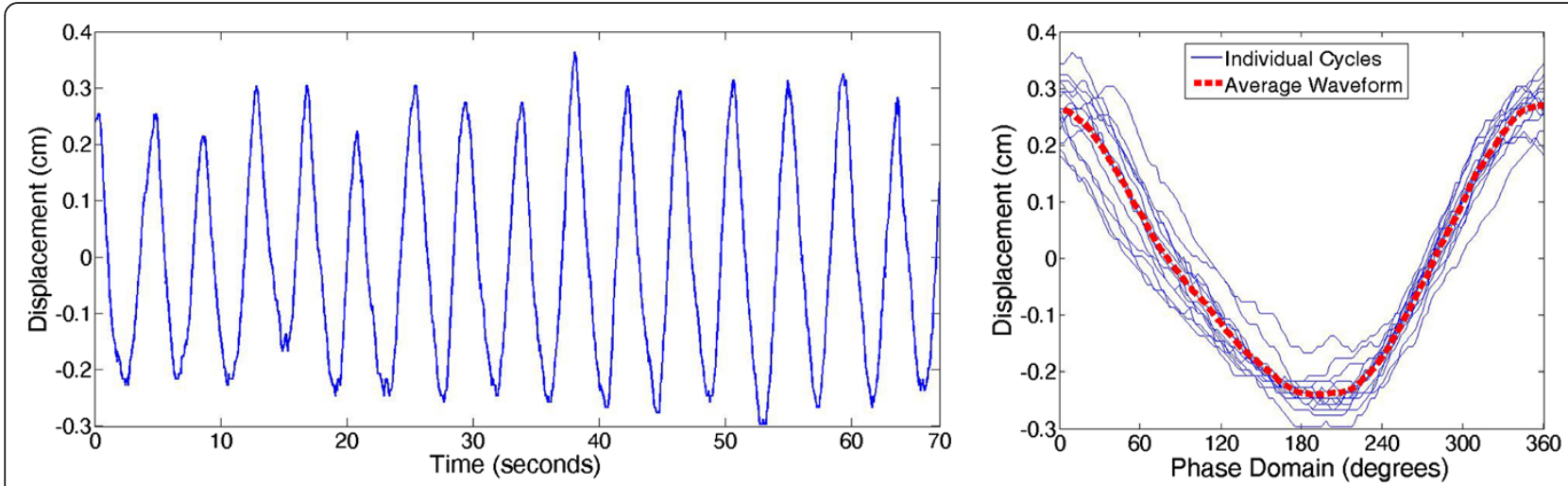

Fig. 4 Example of breathing motion trace (left) then separated into individual cycles with the average waveform shown as the red dashed curve (right) 
the similarity between edge slices at adjacent couch positions $A$ and $B$; the edge similarity between slice $A$ and slice $B$ is expressed by the normalised correlation coefficient (NCC). Deviations from standard NCC, representing normal anatomical changes between edge slices, signify the presence of an image artefact. Cui, et al., (2012) reported good agreement of their method with the assessment of two observers.

\section{Discussion}

This clinical trial marks an important milestone in breathing guidance studies as it will be the first randomised, controlled trial providing the most comprehensive evaluation of the clinical impact of breathing guidance on cancer radiation therapy to date. Based on the structure of previous investigations, and taking into consideration the increase in scope of this study, the authors have designed a multiinstitutional, randomised, phase II, stratified clinical trial to test the hypothesis that audiovisual biofeedback breathing guidance will significantly improve breathing regularity and reduce medical imaging errors for lung cancer patients undergoing imaging and treatment procedures during radiotherapy. While patients will be stratified by treating institution and for treatment intent, the study is not powered for formal comparisons between the these stratified groups; estimates from the current proposed study of the proportion of patients which do not experience irregular breathing will provide information as to whether further investigation is warranted. Further to this, objectives such as determining the indications and contraindications for the use of audiovisual biofeedback, evaluation of patient experience, radiation toxicity occurrence and severity, and clinician confidence will shed light on the design of future phase III clinical trials.

\section{Abbreviations}

AV biofeedback: Audiovisual biofeedback; HREC: Human Research Ethics Committee; PET: Positron Emission Tomography; MRI: Magnetic resonance imaging; ANZCTR: Australian New Zealand Clinical Trials Registry; RPM: Real-time position management; IGRT: Image-guided radiotherapy; QA: Quality assurance; TAM: Technology acceptance model; ECOG score: Eastern cooperative oncology group score; VCU: Virginia Commonwealth University.
}

\section{Competing interests}

This trial is funded by a National Health and Medical Research Council (NHMRC) Development Grant (application ID: 1093186). Paul Keall is one of the inventors of US patent \# 7955270, and Paul Keall, Sean Pollock, Ricky O'Brien and Kuldeep Makhija are shareholders of Respiratory Innovations, an Australian company that is developing a device to improve breathing stability. No funding or support was provided by Respiratory Innovations.

\section{Authors' contributions}

SP drafted the manuscript, collects and analyses the clinical trial data. $\mathrm{RO}$ developed the software for the intervention used in the trial and is leading the department-specific secondary objectives (4) pertaining to system robustness and fault reporting. KM also developed the AV biofeedback software tailored for clinical use, in additional to performing fault reporting for the department specific secondary objectives (4) pertaining to system robustness and fault reporting. FHJ, JL, AR, TE, RT, and RYA are identifying and recruiting study participants as well as satisfying patient-specific secondary objective (7) pertaining to reporting patient toxicity outcomes. VG performed the power calculation and determined the sample size for the clinical trial; VG is also performing the participant randomisation of the clinical trial. PK conceived the clinical trial and participated in the design of the clinical trial. All authors read and approved the final manuscript.

\section{Acknowledgements}

This trial is funded by a National Health and Medical Research Council (NHMRC) Development Grant (application ID: 1093186)

\section{Author details}

${ }^{1}$ Radiation Physics Laboratory, Sydney Medical School, The University of Sydney, Sydney, NSW, Australia. ${ }^{2}$ Central Coast Cancer Centre, Gosford Hospital, Gosford, NSW, Australia. ${ }^{3}$ Department of Radiation Oncology, Calvary Mater Newcastle, Newcastle, NSW, Australia. ${ }^{4}$ Department of Radiation Oncology, Canberra Hospital, Canberra, ACT, Australia. ${ }^{5}$ Department of Radiation Oncology, Chris O'Brien Lifehouse, Sydney, NSW, Australia. ${ }^{6}$ Department of Radiation Oncology, Northern Sydney Cancer Centre, Sydney, NSW, Australia. ${ }^{7}$ Radiation Oncology Network, Crown Princess Mary Cancer Centre, Westmead Hospital, Sydney, NSW, Australia. ${ }^{8}$ Department of Radiation Oncology, Nepean Cancer Care Centre, Sydney, NSW, Australia. ${ }^{9}$ University of Sydney NHMRC Clinical Trials Centre, Sydney, NSW, Australia.

Received: 8 May 2015 Accepted: 9 June 2015

Published online: 18 July 2015

\section{References}

1. Machtay M, Bae K, Movsas B, Paulus R, Gore EM, Komaki R, et al. Higher biologically effective dose of radiotherapy is associated with improved outcomes for locally advanced non-small cell lung carcinoma treated with chemoradiation: an analysis of the Radiation Therapy Oncology Group. Int J Radiat Oncol* Biol* Phys. 2010(1):425-34.

2. Marks LB, Bentzen SM, Deasy JO, Kong FMS, Bradley JD, Vogelius IS, et al. Radiation dose-volume effects in the lung. Int J Radiat Oncol, Biol, Phys. 2010;76(3):S70-S6.

3. Hugo GD, Campbell J, Zhang T, Yan D. Cumulative lung dose for several motion management strategies as a function of pretreatment patient parameters. Int J Radiat Oncol, Biol, Phys. 2009;74(2):593-601.

4. Keall PJ, Mageras GS, Balter JM, Emery RS, Forster KM, Jiang SB, et al. The management of respiratory motion in radiation oncology report of AAPM Task Group 76. Med Phys. 2006:33(10):3874-900.

5. Vedam S, Keall P, Docef A, Todor D, Kini V, Mohan R. Predicting respiratory motion for four-dimensional radiotherapy. Med Phys. 2004;31:2274.

6. Kocak Z, Evans ES, Zhou SM, Miller KL, Folz RJ, Shafman TD et al. Challenges in defining radiation pneumonitis in patients with lung cancer. International Journal of Radiation Oncology* Biology* Physics. 2005;62(3):635-8.

7. Wong JW, Sharpe MB, Jaffray DA, Kini VR, Robertson JM, Stromberg JS et al. The use of active breathing control $(A B C)$ to reduce margin for breathing motion. International Journal of Radiation Oncology* Biology* Physics. 1999;44(4):911-9

8. Seppenwoolde Y, Shirato H, Kitamura K, Shimizu S, van Herk M, Lebesque JV et al. Precise and real-time measurement of 3D tumor motion in lung due to breathing and heartbeat, measured during radiotherapy. Int J Radiat Oncol Biol Phys. 2002;53(4):822-34.

9. Vedam SS, Kini VR, Keall PJ, Ramakrishnan V, Mostafavi H, Mohan R. Quantifying the predictability of diaphragm motion during respiration with a noninvasive external marker. Med Phys. 2003;30(4):505-13.

10. Wong JW, Sharpe MB, Jaffray DA, Kini VR, Robertson JM, Stromberg JS, et al. The use of active breathing control $(A B C)$ to reduce margin for breathing motion. Int J Radiat Oncol, Biol, Phys. 1999;44(4):911-9.

11. Seppenwoolde $Y$, Shirato H, Kitamura K, Shimizu S, van Herk M, Lebesque $\mathrm{J}$, et al. Precise and real-time measurement of 3D tumor motion in lung due to breathing and heartbeat, measured during radiotherapy. Int J Radiat Oncol, Biol, Phys. 2002;53(4):822-34

12. Vedam SS, Kini VR, Keall PJ, Ramakrishnan V, Mostafavi H, Mohan R. Quantifying the predictability of diaphragm motion during respiration with a noninvasive external marker. Med Phys. 2003;30(4):505-13.

13. Venkat RB, Sawant A, Suh Y, George R, Keall PJ. Development and preliminary evaluation of a prototype audiovisual biofeedback device 
incorporating a patient-specific guiding waveform. Phys Med Biol. 2008;53(11):N197-208. doi:10.1088/0031-9155/53/11/N01.

14. Abdelnour AF, Nehmeh SA, Pan T, Humm JL, Vernon P, Schoder $H$, et al. Phase and amplitude binning for 4D-CT imaging. Phys Med Biol. 2007:52(12):3515-29.

15. Yamamoto T, Langner U, Loo Jr BW, Shen J, Keall PJ. Retrospective analysis of artifacts in four-dimensional ct images of 50 abdominal and thoracic radiotherapy patients. Int J Radiat Oncol, Biol, Phys. 2008;72:1250. doi:10.1016/j.jijobp.2008.06.1937.

16. Lu W, Parikh PJ, Hubenschmidt JP, Bradley JD, Low DA. A comparison between amplitude sorting and phase-angle sorting using external respiratory measurement for 4D CT. Med Phys. 2006;33(8):2964-74.

17. Mutaf YD, Antolak JA, Brinkmann DH. The impact of temporal inaccuracies on 4DCT image quality. Med Phys. 2007;34(5):1615-22.

18. Pan T, Lee TY, Rietzel E, Chen GT. 4D-CT imaging of a volume influenced by respiratory motion on multi-slice CT. Med Phys. 2004;31(2):333-40.

19. Rietzel E, Chen GT, Doppke KP, Pan T, Choi NC, Willett CG. 4D computed tomography for treatment planning. Int J Radiat Oncol, Biol, Phys. 2003;57(2 Suppl):S232-3.

20. Cui G, Gopalan S, Yamamoto T, Berger J, Maxim PG, Keall PJ. Commissioning and quality assurance for a respiratory training system based on audiovisual biofeedback. J Appl Clin Med Phys. 2010;11(4):3262.

21. Kim T, Pollock S, Lee D, O'Brien R, Keall P. Audiovisual biofeedback improves diaphragm motion reproducibility in MRI. Med Phys. 2012;39:6921.

22. Yang J, Yamamoto T, Cho B, Seo Y, Keall PJ. The impact of audio-visual biofeedback on 4D PET images: results of a phantom study. Med Phys. 2012;39(2):1046-57. doi:10.1118/1.3679012.

23. George R, Chung TD, Vedam SS, Ramakrishnan V, Mohan R, Weiss E, et al. Audio-visual biofeedback for respiratory-gated radiotherapy: impact of audio instruction and audio-visual biofeedback on respiratory-gated radiotherapy. Int J Radiat Oncol, Biol, Phys. 2006;65(3):924-33.

24. Lee D, Greer P, Arm J, Keall P, Kim T, editors. Audiovisual biofeedback improves image quality and reduces scan time for respiratory-gated 3D MRI. J Phys: Conf Ser. 2014;489(ICCR 2013):012033.

25. Davis FD. Perceived usefulness, perceived ease of use, and user acceptance of information technology. MIS Q. 1989;13(3):319-40.

26. Davis FD, Bagozzi RP, Warshaw PR. User acceptance of computer technology: a comparison of two theoretical models. Manag Sci. 1989;35(8):982-1003.

27. Treasure T, MacRae KD. Minimisation: the platinum standard for trials? BMJ. 1998;317(7155):362-3.

28. Scott NW, McPherson GC, Ramsay CR, Campbell MK. The method of minimization for allocation to clinical trials: a review. Control Clin Trials. 2002;23(6):662-74.

29. George R, Ramakrishnan V, Siebers JV, Chung TD, Keall PJ. Investigation of patient, tumour and treatment variables affecting residual motion for respiratory-gated radiotherapy. Phys Med Biol. 2006;51(20):5305-19. doi:10.1088/0031-9155/51/20/015

30. Worm ES, Høyer M, Fledelius W, Hansen AT, Poulsen PR. Variations in magnitude and directionality of respiratory target motion throughout full treatment courses of stereotactic body radiotherapy for tumors in the liver. Acta Oncol. 2013;52(7):1437-44.

31. Shah AP, Kupelian PA, Waghorn BJ, Willoughby TR, Rineer JM, Mañon RR, et al. Real-time tumor tracking in the lung using an electromagnetic tracking system. Int J Radiat Oncol, Biol, Phys. 2013;86(3):477-83.

32. vsan Herk M, Remeijer P, Rasch C, Lebesque JV. The probability of correct target dosage: dose-population histograms for deriving treatment margins in radiotherapy. Int J Radiat Oncol, Biol, Phys. 2000;47(4):1121-35.

33. Simon R. Optimal two-stage designs for phase II clinical trials. Control Clin Trials. 1989;10(1):1-10.

34. Ruan D, Fessler JA, Balter JM, Sonke J-J. Exploring breathing pattern irregularity with projection-based method. Med Phys. 2006;33(7):2491-9.

35. Cui G, Jew B, Hong JC, Johnston EW, Loo Jr BW, Maxim PG. An automated method for comparing motion artifacts in cine four-dimensional computed tomography images. J Appl Clin Med Phys. 2012;13(6):3838.

36. An S, Yeo I, Jung J, Suh H, Lee KJ, Choi J, et al. The effect of breathing biofeedback on breathing reproducibility and patient's dose in respiration-gated radiotherapy. Progress in Medical Physics. 2013;24(3):135-9.

37. Pollock S, Lee D, Keall P, Kim T. Audiovisual biofeedback improves motion prediction accuracy. Med Phys. 2013;40:041705.
38. Steel H, Pollock S, Lee D, Keall P, Kim T. The internal-external respiratory motion correlation is unaffected by audiovisual biofeedback. Australas Phys Eng Sci Med. 2014;37(1):1-6.

39. Lu W, Neuner GA, George R, Wang Z, Sasor S, Huang X, et al. Audio-visual biofeedback does not improve the reliability of target delineation using maximum intensity projection in 4-dimensional computed tomography radiation therapy planning. Int J Radiat Oncol, Biol, Phys. 2014;88(1):229-35.

40. Lee D, Greer P, Arm J, Hunter P, Pollock S, Makhija K, et al. SU-EJ-29: Audiovisual biofeedback improves tumor motion consistency for lung cancer patients. Med Phys. 2014;41(6):161.

\section{Submit your next manuscript to BioMed Central and take full advantage of:}

- Convenient online submission

- Thorough peer review

- No space constraints or color figure charges

- Immediate publication on acceptance

- Inclusion in PubMed, CAS, Scopus and Google Scholar

- Research which is freely available for redistribution

Submit your manuscript at www.biomedcentral.com/submit 